\title{
Hydrolytic Metabolism of Cyanopyrrolidine DPP-4 Inhibitors Mediated by Dipeptidyl Peptidases ${ }^{\mathbb{}}$
}

\author{
Fandi Kong, Xiaoyan Pang, Jihui Zhao, Pan Deng, Mingyue Zheng, Dafang Zhong, \\ and Xiaoyan Chen
}

Shanghai Institute of Materia Medica, Chinese Academy of Sciences, Shanghai, P.R. China (F.K., X.P., P.D., M.Z., D.Z., X.C.); University of Chinese Academy of Sciences, Beijing, P.R. China (F.K., M.Z., D.Z., X.C.); and School of Life Science and Technology, Shanghai Tech University, Shanghai, P.R. China (J.Z.)

Received September 25, 2018; accepted December 5, 2018

\section{ABSTRACT}

Nitrile group biotransformation is an unusual or minor metabolic pathway for most nitrile-containing drugs. However, for some cyanopyrrolidine dipeptidyl peptidase 4 (DPP-4) inhibitors (vildagliptin, anagliptin, and besigliptin, but not saxagliptin), the conversion of nitrile group into carboxylic acid is their major metabolic pathway in vivo. DPP-4 was reported to be partly involved in the metabolism. In our pilot study, it was also observed that saxagliptin, a DPP-4 specific inhibitor, decreased the plasma exposures of besigliptin carboxylic acid in rats by only $20 \%$. Therefore, it is speculated that some other enzymes may participate in nitrile group hydrolysis. After incubating gliptins with the cytosol, microsomes, and mitochondria of liver and kidney, carboxylic acid metabolites could all be formed. In recombinant DPP family such as DPP-4, DPP-2, DPP-8, DPP-9, and fibroblast activation protein- $\alpha$, more hydrolytic metabolites were found. Among them, DPP-2 had the highest hydrolytic capacity besides DPP-4, and the DPP-4 inhibitor saxagliptin and DPP-2 inhibitor AX8819 can both inhibit the hydrolysis of gliptins. Western blot results showed that DPP-2 and DPP-4 existed in the aforementioned subcellular organelles at varying amounts. In rats, AX8819 decreased the plasma exposures of besigliptin carboxylic acid by $\mathbf{4 0} \%$. The amide intermediates of gliptins were detected in vivo and in vitro. When the amide derivatives of gliptins were incubated with DPP-4, they were completely hydrolyzed at a rate far more than that from the parent drug, including saxagliptin-amide. Therefore, it was proposed that gliptins, except saxagliptin, were initially hydrolyzed to their amides by DPPs, which was the rate-limiting step in generating the carboxylic end product.

\section{Introduction}

The nitrile $(\mathrm{CN})$ group is introduced as an efficacious pharmacophore into the increasing number of therapeutic drugs. It can enhance the selectivity and binding affinity of these drugs to target proteins via hydrogen bond, covalent, polar, and $\pi-\pi$ interactions. Moreover, nitrile is introduced to block metabolically labile sites because nitrile biotransformation is rather rare and, when observed, is a minor metabolic pathway (Fleming et al., 2010). However, the nitrile group is not stable as it was expected for cyanopyrrolidine dipeptidyl peptidase 4 (DPP-4) inhibitors, namely, vildagliptin (He et al., 2009a) (Novartis, Basel, Switzerland), anagliptin (Furuta et al., 2013) (Sanwa Kagaku Kenkyusho Co., Ltd., Nagoya, Japan), and besigliptin (Jiangsu Hansoh Pharmaceutical Co., Ltd., Lianyungang, China, under clinical phases) (Fig. 1). The hydrolysis of the nitrile group to a carboxyl group is their major metabolic pathway in vivo. However, there is an obvious exception to this: the

This research was financially supported by the National Natural Science Foundation of China [Grant 81573500] and the Strategic Priority Research Program of the Chinese Academy of Sciences [Grant XDA 12050306].

F.K. and X.P. contributed equally to this work.

The authors declare no conflicts of interest.

https://doi.org/10.1124/dmd.118.084640.

SThis article has supplemental material available at dmd.aspetjournals.org. nitrile group of saxagliptin, a cyanopyrrolidine DPP-4 inhibitor on the market (Bristol-Myers Squibb, New York, NY), cannot be hydrolyzed (Su et al., 2012).

It has been reported that DPP-4 could mediate the $\mathrm{CN}$ hydrolysis of vildagliptin (He et al., 2009a; Asakura et al., 2015) and anagliptin (Furuta et al., 2013). However, in DPP-4-knockout rats, the formation of vildagliptin carboxylic acid $(\mathrm{COOH})$ metabolite was only decreased by approximately $20 \%$. Therefore, it is speculated that some other enzymes may participate in CN hydrolysis. DPP-4 (EC 3.4.14.5), also known as $\mathrm{CD} 26$, is a serine peptidase that cleaves off $\mathrm{N}$-terminal dipeptides containing proline, hydroxyproline, or alanine at the penultimate position, such as glucagon-like peptide-1 and glucose-dependent insulinotropic polypeptide (Gautier et al., 2005). DPP-4 activity and/or structure homolog proteins include some other DPPs, such as DPP-2, DPP-8, DPP-9, and fibroblast activation protein- $\alpha$ (FAP). They all belong to the serine peptidase family with a common catalytic triad of Ser, Asp, and His residues in sequence and share similar substrate specificity (Matteucci and Giampietro, 2009), indicating that they might also have the potential hydrolysis ability of the nitrile group. Some severe side effects caused by DPP-4 inhibitors, such as anemia, thrombocytopenia, splenomegaly, lymphadenopathy, lung histiocytosis, mortality with multiple organ pathology, and gastrointestinal and central nervous system toxicity, were reported to be the probable result of the nonspecific inhibition of DPP-8 and DPP-9 (Matteucci and Giampietro, 2009).

ABBREVIATIONS: AUC, area under the plasma concentration-time curve; BCA, bicinchoninic acid; $\mathrm{CN}$, nitrile; $\mathrm{COOH}$, carboxylic acid; DPP, dipeptidyl peptidase; ESI+, positive electrospray ionization; FAP, fibroblast activation protein- $\alpha$; LC-MS/MS, liquid chromatography-tandem mass spectrometry; MS, mass spectrometry; $\mathrm{m} / \mathrm{z}$, mass-to-charge ratio; TOF, time of flight; UPLC, ultra-performance liquid chromatography. 
<smiles>N#C[C@@H]1CCCN1C(=O)CN[C@]12CC3CC(C[C@](O)(C3)C1)C2</smiles>

Vildagliptin<smiles>Cc1cc2ncc(C(=O)NCC(C)(C)NCC(=O)N3CCC[C@H]3C#N)cn2n1</smiles>

Anagliptin<smiles>CN(C)C(=O)N1C[C@H]2C[C@](C)(NCC(=O)N3C[C@@H](F)C[C@H]3C#N)C[C@H]2C1</smiles>

Besigliptin

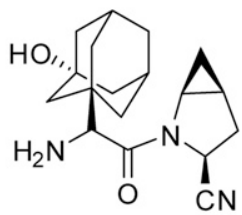

Saxagliptin<smiles>NCC[C@H](N)C(=O)N1CC[C@@H]2CCC[C@H]21</smiles>

AX8819

Fig. 1. Structures of typical cyanopyrrolidine DPP-4 inhibitors and AX8819.

Thus, to avoid off-target effects in the development of DPP-4 inhibitors, DPP-2, DPP-8, DPP-9, and FAP are often selected for screening specific DPP-4 inhibitors. In the present study, the roles of other dipeptidyl peptidases except DPP-4 were investigated during CN hydrolysis metabolism of gliptins. Moreover, the possible mechanism of why saxagliptin could not be hydrolyzed was also discussed.

Besigliptin was employed as a model drug to conduct in vivo pharmacokinetic experiments in rats and was combined with vildagliptin, anagliptin, and saxagliptin to investigate the enzymes involved in the hydrolysis of the nitrile group. Besigliptin is a cyanopyrrolidine DPP-4 inhibitor that is in clinical development. Its main metabolite in humans is also the besigliptin- $\mathrm{COOH}$ metabolite hydrolyzed from the nitrile group. The plasma exposure of besigliptin-COOH was about 8-fold of the parent drug after an oral dose of besigliptin in humans (unpublished data).

\section{Materials and Methods}

Chemicals and Reagents. Besigliptin, its carboxylic acid metabolite, and its amide derivative were all kindly provided by Jiangsu Hansoh Pharmaceutical Co., Ltd. Saxagliptin and vildagliptin were purchased from Meilun Biology Technology Co., Ltd. (Dalian, China). Anagliptin was obtained from Chengdu Novel Biochemical Technology Co., Ltd. (Chengdu, China). Amide derivatives of saxagliptin and vildagliptin were purchased from Phystandard Bio-Tech Co., Ltd. (Shenzhen, China). AX8819 (Fig. 1) was synthesized as previously described (Danilova et al., 2007). Tris was purchased from Sangon Biotech Co., Ltd. (Shanghai, China). The bicinchoninic acid (BCA) protein assay kit was obtained from Beyotime (Shanghai, China). Human liver homogenate, cytosol, mitochondria, microsomes, and kidney homogenate were purchased from BD Gentest (Woburn, MA). Recombinant DPP-4 and DPP-2 were obtained from GenScript Corporation (Nanjing, China). DPP-8, DPP-9, and FAP were kindly provided by Dr. Jingya Li at the National Center for Drug Screening (Shanghai, China). All solvents for liquid chromatography-tandem mass spectrometry (LC-MS/MS) analysis were of high-performance liquid chromatography grade (Merck, Darmstadt, Germany). Other reagents were of analytical grade.

Isolation of Rat Hepatic and Renal Subcellular Organelles. Male SpragueDawley rats weighing 180-220 g were sacrificed via exsanguination of the abdominal aorta under anesthesia, and the livers or kidneys were promptly removed. One portion of rat liver or kidney sample was homogenized with a 5-fold volume of ice-cold phosphate buffer $(250 \mathrm{mM}$ sucrose, $10 \mathrm{mM}$ Tris-HCl, $1 \mathrm{mM}$ EDTA-2Na, $\mathrm{pH}$ 7.4) before centrifuging at $600 \mathrm{~g}$ for 10 minutes at $4^{\circ} \mathrm{C}$ to remove tissue debris and nuclei. The supernatant was spun at $9000 \mathrm{~g}$ for 20 minutes to obtain the $\mathrm{S} 9$ fraction. The resulting pellet comprised mitochondria and was washed once before analysis. The $\mathrm{S} 9$ fraction was further centrifuged at $100,000 \mathrm{~g}$ for 60 minutes at $4^{\circ} \mathrm{C}$ to separate the cytosolic and microsome components. Each pellet was dissolved in adequate homogenization buffer and stored at $-80^{\circ} \mathrm{C}$. The protein content was determined using the BCA protein assay kit.

Subcellular Organelle Incubations. Besigliptin $(0.25 \mu \mathrm{M})$, vildagliptin $(0.50 \mu \mathrm{M})$, and anagliptin $(0.50 \mu \mathrm{M})$ were respectively incubated with different organelles (protein concentration: $4 \mathrm{mg} / \mathrm{ml}$ ) at $37^{\circ} \mathrm{C}$ for 3 hours, including microsomes, mitochondria, and cytosol of rat liver, rat kidney, and human liver. The hydrolysis was also investigated in homogenates (protein concentration: $4 \mathrm{mg} / \mathrm{ml}$ ) of the liver and kidney in rats and humans. The incubation system comprised phosphate-buffered saline $(100 \mathrm{mM}, \mathrm{pH} 7.5)$ with $0.5 \mathrm{mM} \mathrm{MgCl}_{2}$. The time and protein concentration had been investigated in a prestudy to avoid saturation. The total volume was $200 \mu$ l. Reactions were terminated with an equal volume of ice-cold acetonitrile. Samples were stored at $-80^{\circ} \mathrm{C}$ until LC-MS/MS analysis.

Immunoblot Analysis of Kidney and Liver Homogenate and Their Subcellular Organelles. The liver homogenate, mitochondria, microsomes, and cytosol were diluted to the desired protein concentration with 4X lithium dodecyl sulfate buffer after the measurement of protein concentrations using the BCA protein assay kit. Samples were separated by $4 \%-20 \%$ Bis-Tris PAGE (GenScript Corporation) and transferred to a polyvinylidene fluoride membrane. Blots were probed with the primary antibody of 1:100 dilution of rabbit anti-DPP-2 polyclonal antibody (Thermo Fisher Scientific, Cambridge, MA) and 1:1000 dilution of rabbit anti-DPP-4 monoclonal antibody (Abcam, Cambridge, MA). $\beta$-Actin (1:500 dilution) (Santa Cruz Biotechnology, Dallas, TX) was selected as the loading control for homogenates. No proper endogenous protein was evenly distributed in the cytosol, mitochondria, and microsomes. Thus, the same loading amounts were roughly ensured by loading the same amount of proteins measured through the BCA method. The signal was visualized by adding chemiluminescent agent (Millipore, Billerica, MA) after incubating with a 1:2000 dilution of the secondary horseradish peroxidase-labeled antibody (goat anti-rabbit $\operatorname{IgG}$ ) (KPL, Gaithersburg, MD).

Hydrolysis of Gliptins and Amide Derivatives in Recombinant DPPs. Besigliptin, vildagliptin, anagliptin, and saxagliptin, and their amide derivatives, except anagliptin (terminal concentration: $100 \mu \mathrm{M}$ ), were incubated with DPP-4, DPP-2, DPP-8, DPP-9, and FAP, respectively, in phosphate-buffered saline with their optimum $\mathrm{pH}$ (DPP-4: $\mathrm{pH}$ 7.5; DPP-2: $\mathrm{pH} 5.5$; DPP-8/9 and FAP: $\mathrm{pH}$ 8.0) at $37^{\circ} \mathrm{C}$ for 3 hours. The concentration of DPPs was set at the same hydrolytic activity of serine according to the National Center for Drug Screening. Each treatment was performed in duplicate.

To compare the reaction rate of gliptin as substrate with amide derivative as substrate, besigliptin $(100 \mu \mathrm{M})$ and its amide $(100 \mu \mathrm{M})$ were separately incubated at $37^{\circ} \mathrm{C}$ in the recombinant DPP-4 $(5.00 \mu \mathrm{g} / \mathrm{ml})$ incubation system at designated time points. The reaction rates from three gliptin amide derivatives to the corresponding carboxylic acid metabolites were further compared. Amide derivatives $(50.0 \mu \mathrm{M})$ of besigliptin, vildagliptin, and saxagliptin were separately incubated with DPP-4 $(5.00 \mu \mathrm{g} / \mathrm{ml})$ at $37^{\circ} \mathrm{C}$ and designated time points to investigate the rate of carboxylic acid formation. Reactions were terminated with 


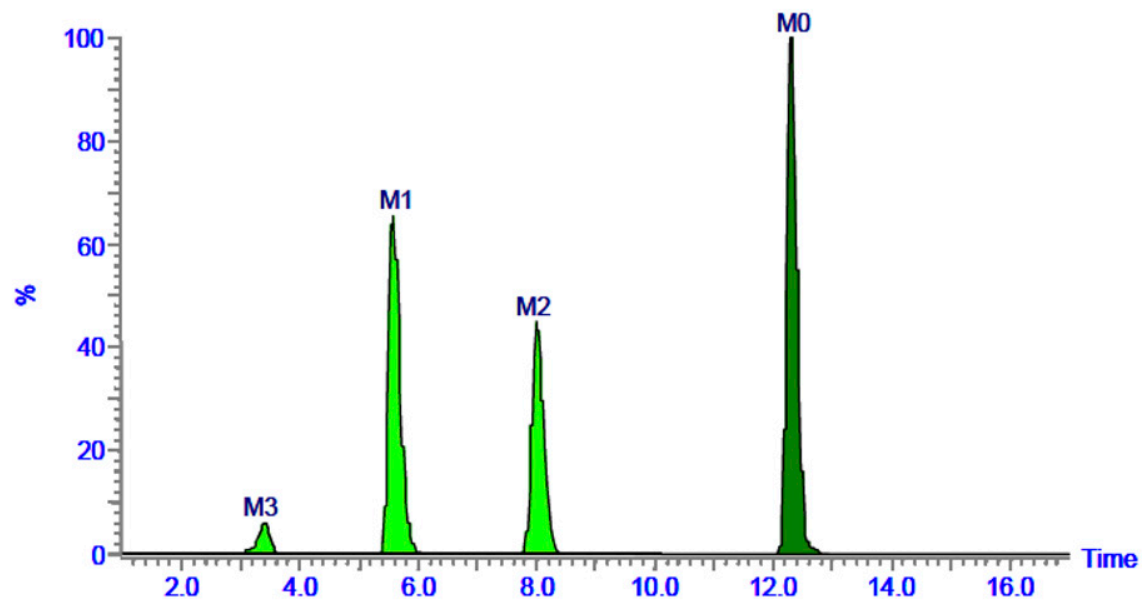

B Combined Metabolite Peaks (Found Expected Peaks only) [Analyte]

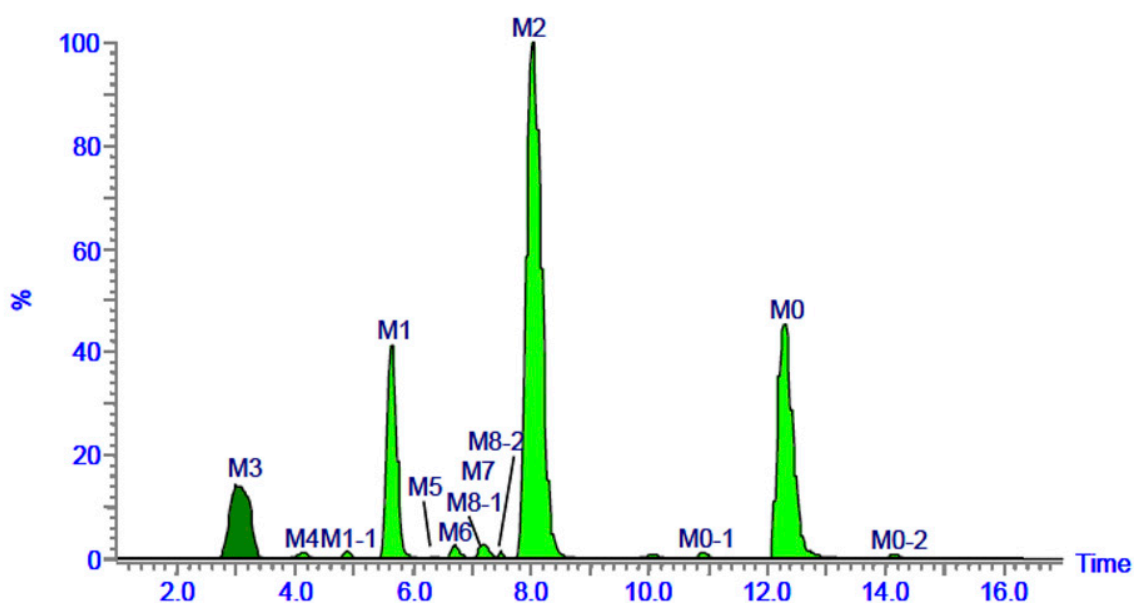

Fig. 2. Extracted ion chromatograms of metabolites in human plasma sample 2 hours postdose (A) and $0-24$ hour urine sample (B) after $100 \mathrm{mg}$ oral dose of besigliptin. an equal volume of ice-cold acetonitrile. Samples were stored at $-80^{\circ} \mathrm{C}$ until ultra-performance liquid chromatography (UPLC)/TripleTOF $5600^{+}$(Applied Biosystems, Ontario, Canada) mass spectrometry (MS) analysis.

Inhibition of Nitrile Group Hydrolysis in Recombinant DPP-4 and DPP-2. To verify the involvement of DPP-2 and DPP-4 in the hydrolysis of gliptins, besigliptin $(10.0 \mu \mathrm{M})$, vildagliptin $(10.0 \mu \mathrm{M})$, and anagliptin $(10.0 \mu \mathrm{M})$ were separately incubated with recombinant DPP-4 $(5.00 \mu \mathrm{g} / \mathrm{ml})$ and DPP$2(5.00 \mu \mathrm{g} / \mathrm{ml})$ at $37^{\circ} \mathrm{C}$ for 3 hours in the presence or absence of DPP-4 inhibitor, saxagliptin $(50.0 \mu \mathrm{M})$, or DPP-2 inhibitor, AX8819 $(50.0 \mu \mathrm{M})$. Reactions were terminated with an equal volume of ice-cold acetonitrile. Samples were stored at $-80^{\circ} \mathrm{C}$ until LC-MS/MS analysis.

Animal Experiments. All procedures involving animals were performed in accordance with the Guide for the Care and Use of Laboratory Animals of the Shanghai Institute of Materia Medica, Chinese Academy of Sciences. The animals were fasted for 12 hours with free access to water before the experiments. Male Sprague-Dawley rats weighing 180-220 g were acclimatized for at least 7 days before the experiments.

To investigate the effects of saxagliptin (DPP-4 inhibitor) on besigliptin metabolism, rats were randomly divided into control and saxagliptin-treated groups. The saxagliptin-treated rats were orally administered $100 \mathrm{mg} / \mathrm{kg}$ per day of saxagliptin formulated in $0.5 \%$ sodium carboxymethyl cellulose for four successive days, and the control group of rats was orally administered the corresponding vehicle control. At 15 minutes after the last dose, the two groups of rats were orally administered $3 \mathrm{mg} / \mathrm{kg}$ of besigliptin dissolved in $0.5 \%$ sodium carboxymethyl cellulose. Blood samples were collected at 10, 20, and 40 minutes, as well as 1.0, 1.5, 2.0, 3.0, 5.0, 7.0, and 10 hours after besigliptin dosage in tubes containing an anticoagulant (EDTA-2K) and $100 \mu \mathrm{M}$ saxagliptin (to avoid further hydrolysis of besigliptin by DPP-4 in the blood). Plasma samples were centrifuged at $11,000 \mathrm{rpm}$ for 5 minutes at $4^{\circ} \mathrm{C}$ and then stored at $-80^{\circ} \mathrm{C}$ until analysis.

The effects of AX8819 (DPP-2 inhibitor) on besigliptin metabolism were evaluated using the aforementioned method. The dosage of AX8819 was $50 \mathrm{mg} / \mathrm{kg}$, whereas the dosage of besigliptin was $1 \mathrm{mg} / \mathrm{kg}$.

Sample Pretreatment. The samples from the rat pharmacokinetic study were prepared as follows. A $25.0 \mu \mathrm{l}$ aliquot plasma sample and $25.0 \mu \mathrm{l}$ of internal standard $(15.0 \mathrm{ng} / \mathrm{ml} \mathrm{SHR} 116022)$ were mixed with $150 \mu \mathrm{l}$ of acetonitrile. After the samples were vortexed and centrifugated at $14,000 \mathrm{rpm}$ for 5 minutes, the supernatants were used to measure besigliptin and its carboxylic acid by the LC-MS/MS analysis.

The samples from subcellular organelle incubation experiments and inhibition experiments on DPP-2 and DPP-4 were pretreated as follows. A $50.0 \mu \mathrm{l}$ aliquot of samples and $25.0 \mu \mathrm{l}$ of internal standard ( $15.0 \mathrm{ng} / \mathrm{ml}$ SHR116022) were mixed with $125 \mu \mathrm{l}$ of acetonitrile. After vortexing and centrifugation at 14,000 rpm for 5 minutes, the supernatants were used to measure gliptins and their carboxylic acid by the LC-MS/MS method.

Samples from the hydrolysis experiments of gliptins and amide derivatives in recombinant DPPs for verification of the mechanism of hydrolysis were vortexed for 1 minute and centrifuged at $14,000 \mathrm{rpm}$ and $4{ }^{\circ} \mathrm{C}$ for 5 minutes. The supernatants were evaporated to dryness at $40^{\circ} \mathrm{C}$ in a nitrogen steam and then reconstituted in $100 \mu \mathrm{l}$ of water/methanol (90:10, v/v). A $10.0 \mu \mathrm{l}$ aliquot of the 

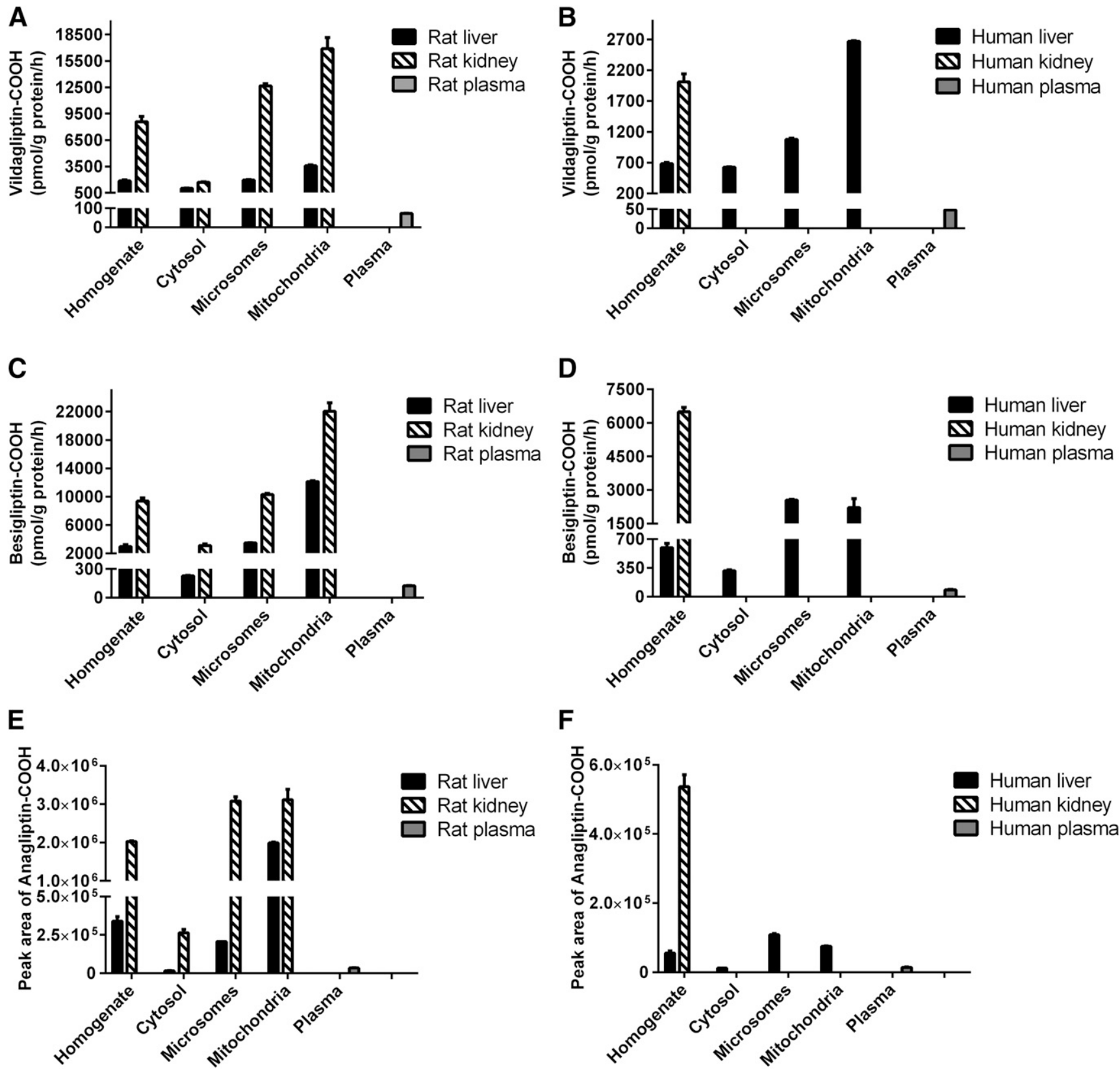

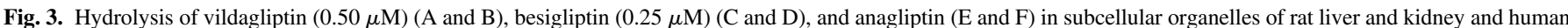
liver $(n=3)$.

reconstituted solution was injected into the UPLC/TripleTOF $5600^{+}$system (Applied Biosystems) for analysis.

UPLC/TripleTOF $\mathbf{5 6 0 0}^{+}$MS Analysis. Metabolite identification was performed using an Acquity UPLC system (Waters Corp., Milford, MA) and a TripleTOF $5600^{+}$system (Applied Biosystems). Chromatographic separation was achieved on UPLC HSS T3 $(100 \mathrm{~mm} \times 2.1 \mathrm{~mm}$ i.d., $1.8 \mu \mathrm{m}$; Waters Corp. $)$ at $40^{\circ} \mathrm{C}$. The mobile phase was a mixture of $5 \mathrm{mM}$ ammonium acetate containing $0.1 \%$ formic acid (A) and acetonitrile (B) at a flow rate of $0.400 \mathrm{ml} / \mathrm{min}$. The gradient elution program began from $1 \% \mathrm{~B}$, was maintained for 1 minute, and then increased linearly to $99 \% \mathrm{~B}$ in the next 15 minutes and maintained for 1 minute; in the next 0.5 minute, the gradient was reduced to $1 \% \mathrm{~B}$ linearly and maintained at $1 \% \mathrm{~B}$ until the gradient was stopped at 20 minutes.

MS detection was performed on the TripleTOF $5600^{+}$mass spectrometer. The mass range was set at mass-to-charge ratio $(\mathrm{m} / \mathrm{z})$ 50-1000, and the other parameters were set as follows: ion spray voltage, $5500 \mathrm{~V}$; declustering potential, $80 \mathrm{~V}$; ion source heater, $550^{\circ} \mathrm{C}$; curtain gas, $35 \mathrm{psi}$; ion source gas $1,60 \mathrm{psi}$; and ion source gas 2, 60 psi. For the TOF (time of flight) MS scans, the collision energy was $10 \mathrm{eV}$; for product scans, the collision energy was $25 \mathrm{eV}$, and the collision energy spread was $15 \mathrm{eV}$. Information-dependent acquisition, together with a real-time multiple mass defect filter, was used to trigger the acquisition of the MS/MS spectra. The compounds were detected in positive electrospray ionization (ESI+) mode. The extracted ions were 384.2411 (besigliptinamide), 385.2251 (besigliptin-COOH), 322.2131 (vildagliptin-amide), 323.1971 (vildagliptin-COOH), 402.2254 (anagliptin-amide), 403.2094 (anagliptin-COOH), 335.1971 (saxagliptin-amide), and 334.2131 (saxagliptin-COOH).

LC-MS/MS Analysis. The parent drugs and their carboxylic acid metabolites were determined on a Shimadzu LC-30AD high-performance liquid chromatography system (Kyoto, Japan) tandem with an API 5500 triplequadrupole MS (Applied Biosystems). The Analyst 1.6.3 software (Applied Biosystems) was used for data acquisition and processing. The mobile phase was a mixture of $5 \mathrm{mM}$ ammonium acetate containing $0.1 \%$ formic acid (A) and acetonitrile (B). 

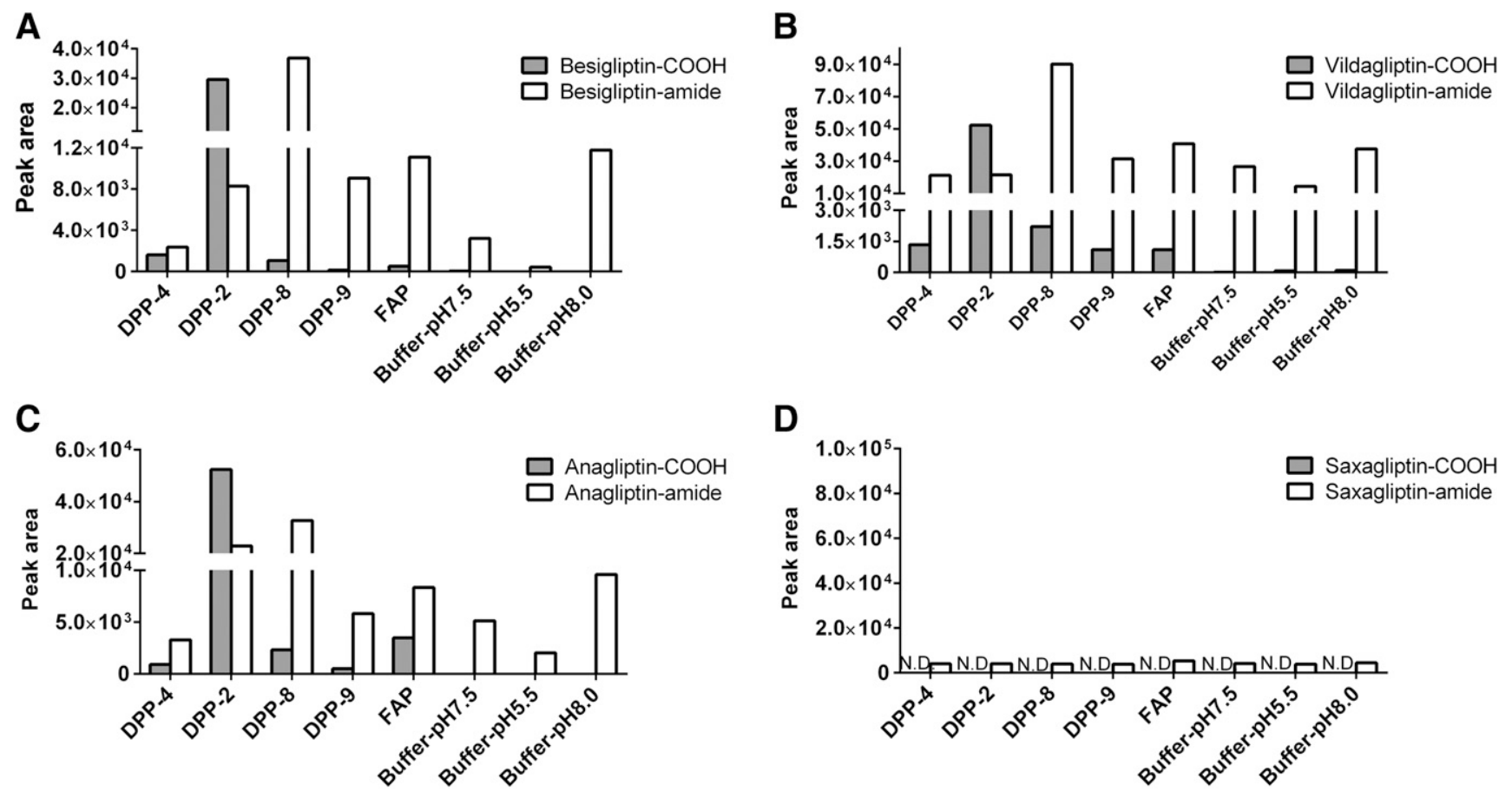

Fig. 4. Hydrolysis of besigliptin (A), vildagliptin (B), anagliptin (C), and saxagliptin (D) in DPP-4, DPP-2, DPP-8, DPP-9, and FAP, and their corresponding incubation buffer. Data are displayed as the mean of two separate samples.

The chromatographic separation of besigliptin and its carboxylic acid metabolite was achieved on Venusil ASB-C18 $(50 \mathrm{~mm} \times 4.6 \mathrm{~mm}$ i.d., $5 \mu \mathrm{m}$; Agela Technologies Inc., Newark, DE) at $40^{\circ} \mathrm{C}$. The mobile phase was set at A and $\mathrm{B}(6: 4, \mathrm{v} / \mathrm{v})$ at a flow rate of $0.65 \mathrm{ml} / \mathrm{min}$. Multiple reactions monitoring $(\mathrm{m} / \mathrm{z}$ $366 \rightarrow 195$ for besigliptin, $\mathrm{m} / z 385 \rightarrow 195$ for besigliptin-COOH, and $\mathrm{m} / z 334 \rightarrow$ 165 for SHR116022 as internal standard) was used in the ESI+ mode with ion spray voltage of $4500 \mathrm{~V}$ and source temperature of $500^{\circ} \mathrm{C}$. The nebulizer, heater, and curtain gases were set to 50,50 , and $20 \mathrm{psi}$, respectively. The standard curve ranges ranged from 1 to $1000 \mathrm{nM}$.

Chromatographic separation of vildagliptin and its carboxylic acid metabolite was achieved on a Luna HILIC HPLC column $(100 \mathrm{~mm} \times 3.0 \mathrm{~mm}$ i.d., $3 \mu \mathrm{m}$; Phenomenex, Inc., Torrance, CA) at $40^{\circ} \mathrm{C}$. The flow rate was $0.80 \mathrm{ml} / \mathrm{min}$. The gradient elution program began from $90 \% \mathrm{~B}$, was maintained for 0.8 minute, and then decreased linearly to $75 \% \mathrm{~B}$ in the next 0.5 minute and maintained for 0.9 minute; in the next 0.1 minute, the gradient was increased to $90 \%$ B linearly and maintained until the gradient was stopped at $4.2 \mathrm{~min}$. Multiple reactions monitoring $(\mathrm{m} / \mathrm{z} 304 \rightarrow 154$ for vildagliptin, $\mathrm{m} / \mathrm{z} 323 \rightarrow 116$ for vildagliptin$\mathrm{COOH}$, and $\mathrm{m} / z, 334 \rightarrow 165$ for SHR116022 as internal standard) was used in the ESI+ mode. Other conditions were the same as previously described.

Chromatographic separation of anagliptin and its carboxylic acid metabolite was achieved on UPLC HSS T3 $(50 \mathrm{~mm} \times 2.1 \mathrm{~mm}$ i.d., $1.8 \mu \mathrm{m}$; Waters Corp. $)$ at $40^{\circ} \mathrm{C}$. The flow was $0.50 \mathrm{ml} / \mathrm{min}$. The gradient elution program began from $90 \%$ $\mathrm{B}$, was maintained for 0.5 minute, and then decreased linearly to $80 \% \mathrm{~B}$ in the next 0.5 minute and maintained for 0.5 minute; in the next 0.5 minute, the gradient was increased to $90 \%$ B linearly and maintained until the gradient was stopped at $4.0 \mathrm{~min}$. Multiple reactions monitoring $(\mathrm{m} / \mathrm{z}, 384 \rightarrow 231$ for anagliptin and $\mathrm{m} / \mathrm{z}$ $403 \rightarrow 231$ for anagliptin- $\mathrm{COOH}$ ) was used in the ESI+ mode. Other conditions were the same as previously described.

Data Analysis. Data are presented as the mean \pm S.D. $(n \geq 3)$ or mean of the duplicate. WinNonlin version 6.1 (Pharsight Corp., Cary, NC) was used to calculate the pharmacokinetic parameters in a noncompartmental model. Student's two-tailed unpaired $t$ test in SPSS version 20.0 (SPSS Inc., Chicago, IL) was used to determine the difference. The level of statistical significance was set at $P<0.05$.

\section{Results}

Identification of Besigliptin Carboxylic Acid and Amide Metabolites in Human Plasma and Urine Samples. Figure 2 show the metabolic profiles in the human plasma sample ( 2 hour postdose) and urine sample (0-24 h) after an oral dose of $100 \mathrm{mg}$ besigliptin. M2 was a major metabolite in both the plasma and urine samples with a protonated molecule at $\mathrm{m} / \mathrm{z}$ 385.225, which has identical chromatographic behavior and product ions with the besigliptin- $\mathrm{COOH}$ standard. The typical ion spectra of M2 (besigliptin-COOH) are shown in Supplemental Fig. 1B. Trace amount of M8-2 was detected in the human urine sample with a protonated molecule at $\mathrm{m} / \mathrm{z} 384.241$, which has the same chromatographic retention and product ions as the besigliptin-amide standard (Supplemental Fig. 1C).

Hydrolysis of Gliptins in Liver and Kidney Subcellular Organelles. Besigliptin, vildagliptin, and anagliptin were hydrolyzed to their corresponding carboxylic acid metabolites in rat and human liver, kidney, and plasma. In addition, the production of carboxylic acid metabolites was in descending order in the hepatic mitochondria, microsomes, and cytosol (Fig. 3). Because it was difficult to obtain human kidney subcellular organelles, only rat kidney organelles were used to investigate the hydrolysis. Carboxylic acid metabolites of besigliptin, vildagliptin, and anagliptin were also produced in the mitochondria, microsomes, and cytosol of rat kidney in descending order (Fig. 3).

Hydrolysis of Gliptins in Recombinant DPPs. The hydrolysis of four gliptins was investigated in DPPs, including DPP-4, DPP-2, DPP-8, DPP-9, and FAP (Fig. 4). Except saxagliptin, the carboxylic acid metabolites of three gliptins were detected in all DPP-included systems (Fig. 4, A-C). Among them, the DPP-2 incubation system generated the most carboxylic acid metabolites, and DPP-9 formed the least. Saxagliptin could not be hydrolyzed in any DPP (Fig. 4D). Additionally, the amide metabolites of gliptins were detected in all of the incubation systems, including the buffer of $\mathrm{pH} 5.5$ and 8.0. Moreover, the amount of amide metabolites formed in alkaline buffer was larger than that formed in acidic buffer. In the buffer with $\mathrm{pH} 5.5$, the produced amide metabolites in the buffer alone were less than those in the buffer with 
A

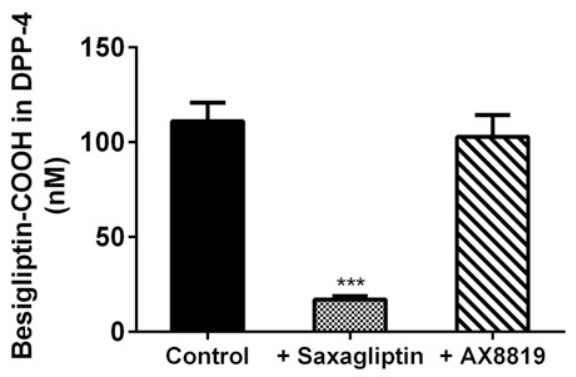

C

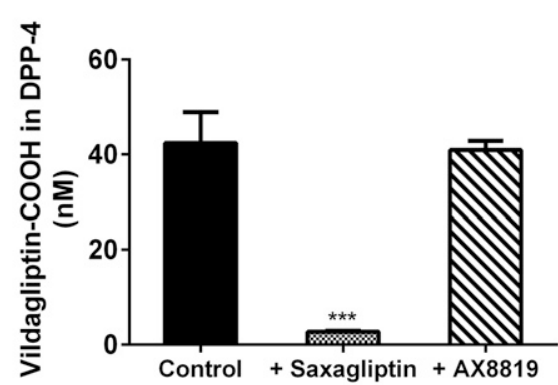

E

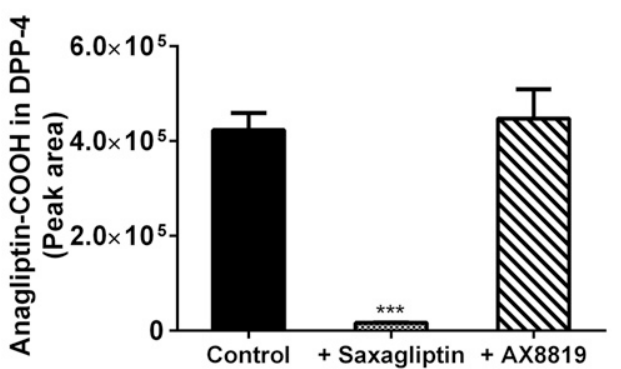

B

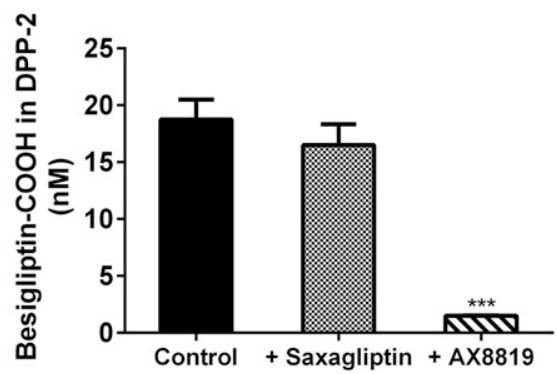

D

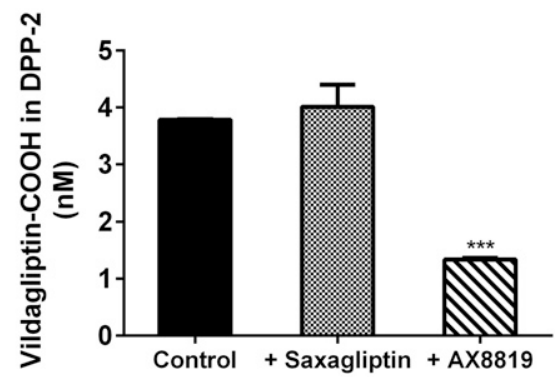

$\mathbf{F}$

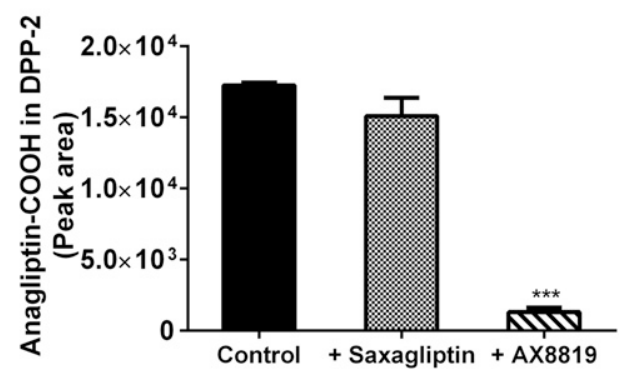

Fig. 5. Hydrolysis of besigliptin $(10 \mu \mathrm{M})$ [(A) DPP-4; (B) DPP-2], vildagliptin $(10 \mu \mathrm{M})$ [(C) DPP-4; (D) DPP-2], and anagliptin (10 $\mu \mathrm{M})$ [(E) DPP-4; (F) DPP-2] in the presence or absence of saxagliptin $(50 \mu \mathrm{M})$ or AX8819 $(50 \mu \mathrm{M})(n=3)$ in phosphate-buffered saline (pH 7.4). $* * * P<0.001$ compared with control.
DPP-2, indicating the DPPs (DPP-2 in particular) were involved in the formation of amide metabolites.

Inhibition of Gliptin Hydrolysis in Recombinant DPP-4 and DPP-2. The effect of selective inhibitors (saxagliptin and AX8819) on the production of carboxylic acid metabolites was investigated. In recombinant human DPP-4, besigliptin, vildagliptin, and anagliptin were hydrolyzed to their carboxylic acid metabolites (Fig. 5, A, C, and E). Saxagliptin almost entirely inhibited the hydrolysis of gliptins, up to $84.6 \%$ for besigliptin, $93.5 \%$ for vildagliptin, and $96.0 \%$ for anagliptin, but AX8819 did not.

Recombinant human DPP-2 was also able to hydrolyze besigliptin, vildagliptin, and anagliptin to their carboxylic acid metabolites, and the hydrolysis was inhibited by AX8819 up to $91.7 \%$ for besigliptin, $64.6 \%$ for vildagliptin, and $92.4 \%$ for anagliptin, but not by saxagliptin (Fig. 5, $\mathrm{B}, \mathrm{D}$, and $\mathrm{F}$ ).

Hydrolysis of Gliptin Amide Derivatives in Recombinant DPPs. Figure 6 shows that the amide derivatives were stable in buffers, but they could be hydrolyzed to carboxylic acid metabolites (including saxagliptin-amide) in the DPP incubation system. Unlike in cases where gliptins were used as the substrates, the amide derivatives that hydrolyzed to carboxylic acid in DPP-2 were less than those in DPP-4, DPP-8, and FAP.

In Fig. 7, the formation rates of besigliptin- $\mathrm{COOH}$ from besigliptin and besigliptin-amide were compared in DPP-4. Besigliptin-amide $(100 \mu \mathrm{M})$ could be hydrolyzed to carboxylic acid completely in 15 minutes. The formation rate from besigliptin-amide to the carboxylic acid (399 $\mathrm{nmol} / \mathrm{mg}$ protein per minute) was 3380 -fold faster than that from besigliptin $(0.118 \mathrm{nmol} / \mathrm{mg}$ protein per minute). The consuming rate of amide into carboxylic acid (Fig. 8) was similar among besigliptin-amide (0.79), vildagliptin-amide (1.21), and saxagliptinamide (1.76).

Distribution of DPP-2 and DPP-4 Protein in the Human/Rat Liver and Kidney or Corresponding Subcellular Organelles. Immunoblot analysis showed that the amount of DPP-2 in rat kidney was much higher than that in rat liver (Fig. 9A). Likely, the abundance of DPP-4 in rat kidney was also more than that in rat liver. However, the abundance of DPP-2 in human liver was comparable with that in human kidney. Different from the distribution of DPP-2 in the human liver and kidney, the abundance of DPP-4 in human kidney was higher than that in human liver.

The subcellular localization of DPP-2 and DPP-4 in the liver and kidney was also detected (Fig. 9B). DPP-2 and DPP-4 were distributed in the mitochondria, microsomes, and cytosol in descending order in rat liver, human liver, and rat kidney.

Pharmacokinetics of Besigliptin. The plasma concentration-time curves of besigliptin and its carboxylic acid metabolite after oral administration of $3 \mathrm{mg} / \mathrm{kg}$ besigliptin to control and saxagliptin-treated rats are shown in Fig. 10. The $\mathrm{AUC}_{0-\mathrm{t}}$ (area under the plasma concentration-time curve) value for besigliptin in saxagliptin-treated rats was $21 \%$ higher than that of control rats. The $\mathrm{AUC}_{0-t}$ value for besigliptin$\mathrm{COOH}$ in saxagliptin-treated rats was $20 \%$ lower than that of control rats.

The pharmacokinetic characters of besigliptin were also changed by AX8819, an inhibitor of DPP-2 (Fig. 11). After an oral administration of $\mathrm{AX8819}$, the $\mathrm{AUC}_{0-\mathrm{t}}$ value for besigliptin was increased to 2.23 -fold, 
A

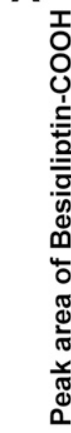

들

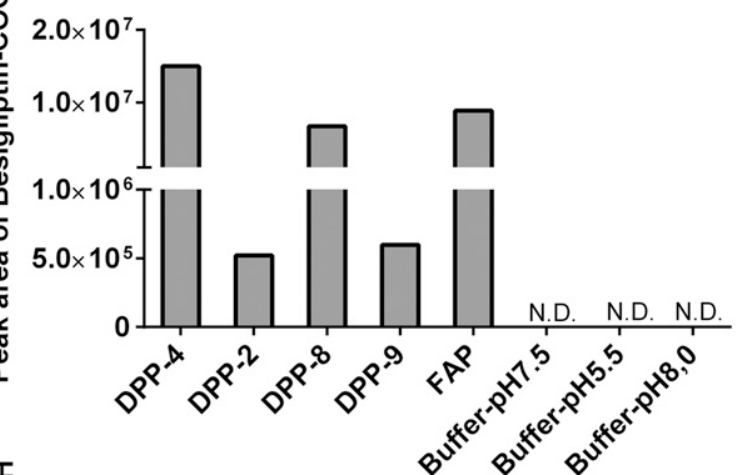

C

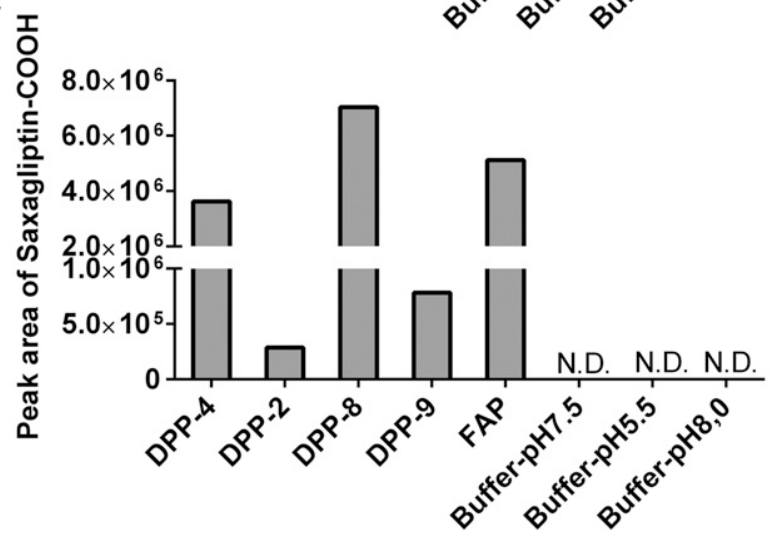

B

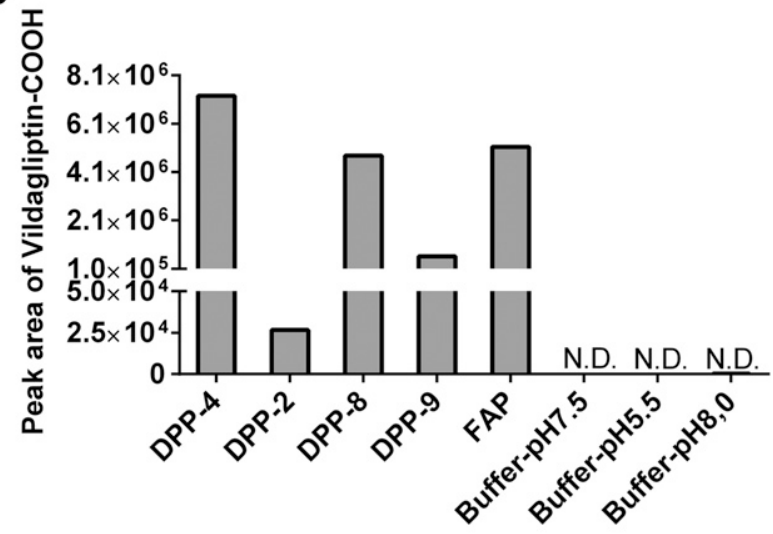

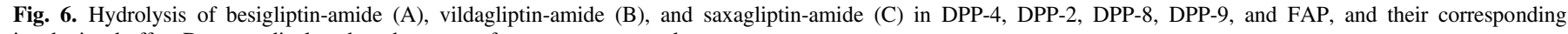
incubation buffer. Data are displayed as the mean of two separate samples.

and the $\mathrm{AUC}_{0-\mathrm{t}}$ value for besigliptin- $\mathrm{COOH}$ was decreased to $62 \%$ compared with control rats.

\section{Discussion}

The nitrile group of cyanopyrrolidine DPP-4 inhibitors vildagliptin, anagliptin, and besigliptin could be hydrolyzed to the carboxylic acid, which were their major metabolites in vivo, accounting for $56.5 \%$ (He et al., 2009a), 29.2\% (Furuta et al., 2013), and 31.6\% (unpublished data) of the administered doses in humans, respectively. However, the nitrile group of saxagliptin could not be hydrolyzed. Thus, it is necessary to confirm the main enzymes involved in the formation of carboxylic acid metabolites of these cyanopyrrolidine DPP-4 inhibitors for proper combination of drugs to avoid drug-drug interactions.
DPP-4 was reported to participate in the partial hydrolysis of vildagliptin (He et al., 2009a; Asakura et al., 2015) and anagliptin (Furuta et al., 2013). Asakura et al. (2015) demonstrated that DPP-4 is expressed in liver microsomes. However, in the prestudy, we found that the carboxylic acid metabolites of gliptins could also be formed by the mitochondria and cytosol of liver and kidney. Therefore, we supposed that some other enzymes participated in the $\mathrm{CN}$ hydrolysis. At the same protein concentration, subcellular organelles of kidney produced more carboxylic acid metabolites than those of the liver in rats and humans, indicating that the kidney is also an important organ for the hydrolytic metabolism of gliptins. The soluble form of DPP-4 was reported to also exist in the serum (Durinx et al., 2000). In Fig. 3, the hydrolysis of the nitrile group in plasma was also observed, but much less than that in the liver and kidney, which would influence the
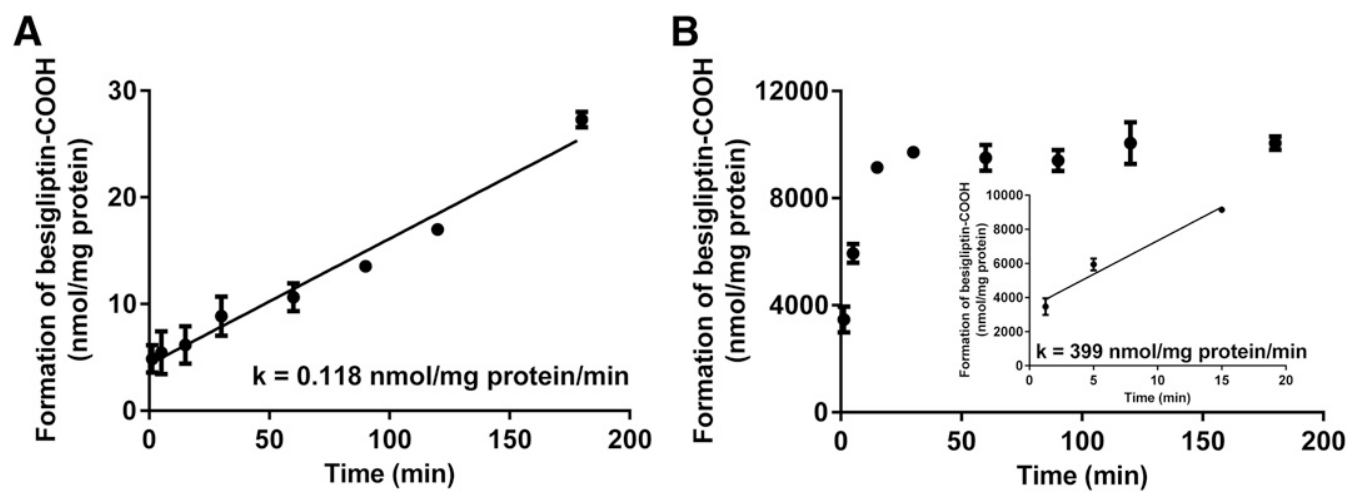

Fig. 7. Formation rate of besigliptin-COOH from besigliptin $(100 \mu \mathrm{M})(\mathrm{A})$ and besigliptin-amide $(100 \mu \mathrm{M})(\mathrm{B})$ in the DPP-4 incubation system $(n=3)$. 


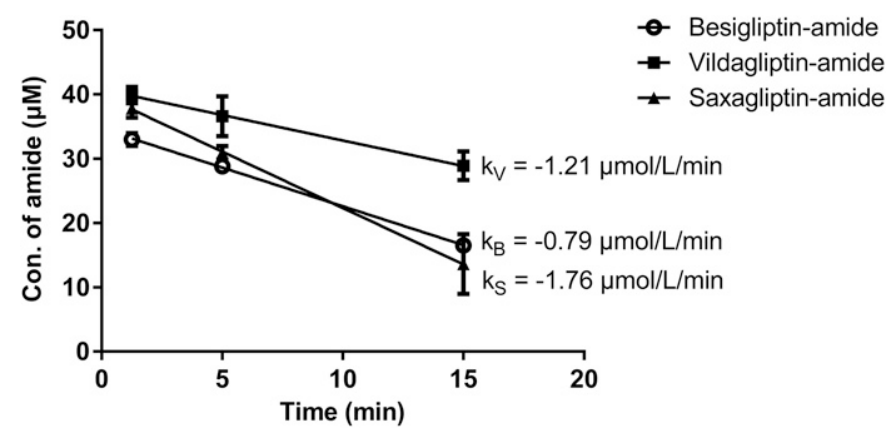

Fig. 8. Formation rate from besigliptin-amide $\left(k_{\mathrm{B}}\right)$, vildagliptin-amide $\left(k_{\mathrm{V}}\right)$, and saxagliptin-amide $\left(k_{\mathrm{S}}\right)$ to their corresponding carboxylic acid $(n=3)$.

detection accuracy of gliptins in the low plasma concentration. In our prestudy, the hydrolysis of besigliptin $(5 \mu \mathrm{M})$ in plasma could be inhibited by saxagliptin $(100 \mu \mathrm{M})$ by more than $90 \%$ (Supplemental Fig. 2), indicating that DPP-4 is the main participated isoform of hydrolysis in plasma. As a result, it is suggested that DPP-4 inhibitors should be added to plasma samples to avoid hydrolysis by the DPP-4 in pharmacokinetic investigations of these cyanopyrrolidine DPP-4 inhibitors.

In DPP-4-knockout rats, the formation of vildagliptin carboxylic acid metabolite was only decreased by approximately $20 \%$ (He et al., 2009b). DPP-4 belongs to the serine peptidase family. Therefore, the hydrolysis activity of its homologous enzymes, including DPP-2, DPP-8, DPP-9, and FAP, to the nitrile group was also investigated. Besides DPP-4, DPP-2 showed the most turnover capacity of the hydrolytic metabolism of gliptins, followed by DPP-8, FAP, and DPP-9 (Fig. 4, A-C). Although the generation of carboxylic acid metabolites in DPP-4, DPP-8, DPP-9 and FAP was similar, it should be noted that the gliptins were all strong DPP-4 inhibitors. In this present study, a high concentration $(100 \mu \mathrm{M})$ of gliptins was used such that the amide concentration could be detected clearly. The high level of gliptins also powerfully inhibited the catalytic ability of DPP-4. When $250 \mathrm{nM}$ besigliptin was incubated in DPPs, only DPP-4 and DPP-2 showed a significant turnover ability of besigliptin to its carboxylic acid, but not the other DPPs (Supplemental Fig. 3). It was suggested that the affinity of gliptins to DPP-2 and DPP-4 was much greater than that to other DPPs. Lower concentrations of gliptins preferred to enter the pocket of DPP-2 and DPP-4, whereas only much higher concentrations of gliptins could get into the pocket of other DPPs. Therefore, considering the concentration of gliptins in vivo was not as high as $100 \mu \mathrm{M}$, DPP-4 and DPP-2 were considered to have a greater contribution to the gliptin hydrolysis.

It was reported that the expression and activity of DPP-8 and DPP-9 are lower than those of DPP-4 in rats, monkeys, and humans (Harstad et al., 2013; Fagerberg et al., 2014); therefore, combined with their limited hydrolysis ability, they were not chosen for the in vitro and in vivo inhibition studies. Considering the stronger hydrolysis activity of DPP-2 and DPP-4 for both low and high concentrations of gliptins, their protein expressions were determined in the liver and kidney subcellular organelles. Western blot results indicated that the expressions of DPP-2 and DPP-4 were higher in the mitochondria and microsomes of rat liver and kidney and human liver. In the cytosol, very low expressions of DPP-2 and DPP-4 (Fig. 9) were found, which also were in accordance with the hydrolytic activity results in the subcellular organelles (Fig. 3).

To further confirm the hydrolysis of gliptins catalyzed by DPP-4 and DPP-2, inhibition experiments were launched in vitro and in vivo. In the DPP-4 incubation system, the hydrolysis of besigliptin, vildagliptin, and anagliptin could be almost completely inhibited by the DPP-4 inhibitor saxagliptin (Fig. 5). However, in rats pretreated with saxagliptin, the $\mathrm{AUC}_{0-\mathrm{t}}$ value of besigliptin-COOH was only decreased by $20 \%$. In the

A

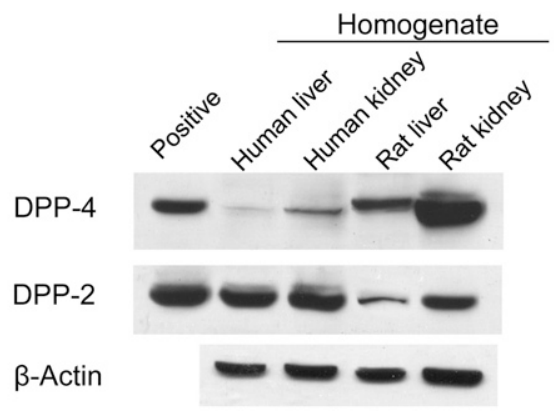

B

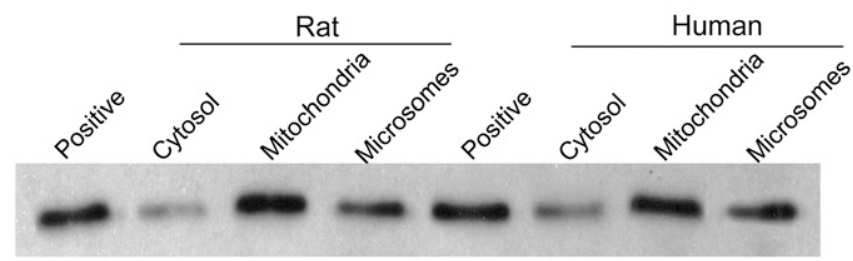

DPP-2 in liver
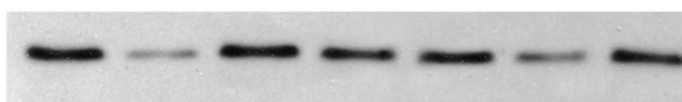

DPP-4 in kidney
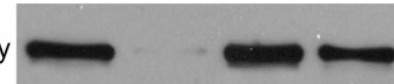

Fig. 9. Immunoblot analysis of DPP-2 and DPP-4 in the rat or human liver and kidney homogenates (A) or corresponding subcellular organelles (B). Homogenates or different intracellular fractions were subjected to $4 \%-20 \%$ Bis-Tris polyacrylamide gel and probed with anti-DPP-2 or anti-DPP-4 antibody. 

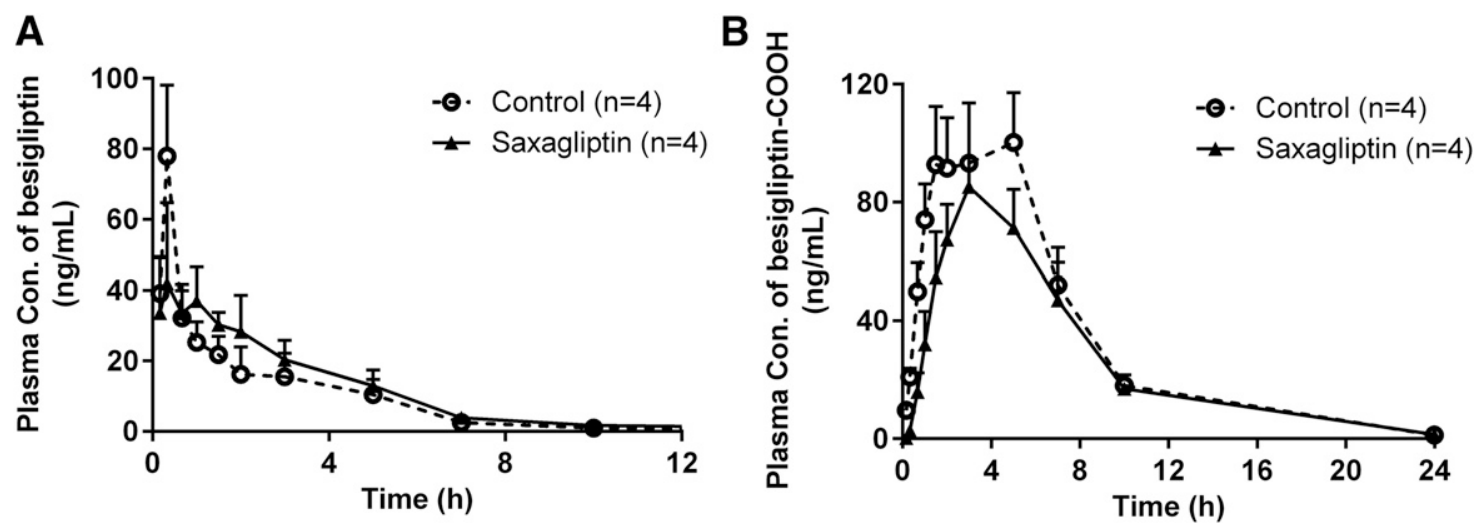

Fig. 10. Mean plasma concentration-time profiles of besigliptin (A) and its carboxylic acid metabolite (B) following oral administration of besigliptin ( $3 \mathrm{mg} / \mathrm{kg}$ ) to control and saxagliptin-treated rats.

DPP-2 incubation system with the physiologic $\mathrm{pH}$, the hydrolysis of three gliptins could be inhibited by the DPP-2 inhibitor AX8819 (Danilova et al., 2007). In the rats pretreated with $\mathrm{AX} 8819$, the $\mathrm{AUC}_{0-\mathrm{t}}$ and $C_{\max }$ values of the besigliptin- $\mathrm{COOH}$ metabolite significantly decreased to $56.7 \%$ and $63.9 \%$, respectively, whereas the $\mathrm{AUC}_{0-\mathrm{t}}$ value of the parent drug increased to 2.23-fold compared with the control rats. Using the docking method, we found that similar to DPP-4, besigliptin is located in the active triad of DPP-2 and bound to DPP-2 through hydrogen bonds to Glu-78, stabilizing the complex. By comparing the score between DPP-2 and DPP-4, we found that the score of DPP- 2 for the metabolism of besigliptin is better than that of DPP-4, suggesting that the binding to DPP-2 is stronger (unpublished data). These results indicated that DPP-2 possibly made a greater contribution to the hydrolysis of gliptins than DPP-4. However, since the content of DPP-2 in vivo has not been confirmed thus far, the contribution of DPP2 needs further research.

In the incubation of besigliptin, vildagliptin, and anagliptin with DPPs, their corresponding amide metabolites could be detected (Fig. 4) and could also be detected in human urine after oral administration of besigliptin (Fig. 2). When the amide metabolite standards were incubated as substrates, a large amount of carboxylic acid metabolites were detected, with the most production in DPP-4, DPP-8, and FAP and the least in DPP-2 and DPP-9 incubation systems (Fig. 6). No carboxylic acid was detected in the buffer without enzymes. Unlike the case with amides as substrates, when the gliptins were used as substrates, carboxylic acid metabolites were generated mainly during DPP-2 incubation, but more amide metabolites were produced in DPP-8 incubation (Fig. 4). Thus, it is supposed that there may be different hydrolysis metabolisms between DPP-2 and other DPPs. Surprisingly different from saxagliptin, the saxagliptin-amide could be hydrolyzed to carboxylic acid by DPPs (Fig. 6). In DPP-4 incubation, the formation rate from amide derivatives to carboxylic acid was much faster than that from gliptins to carboxylic acid (Figs. 7 and 8). These results indicated that DPP-4, DPP-2, and DPP-8 mainly participated in the CN hydrolysis of gliptins, and the formation of amide metabolites was the initial step of hydrolytic metabolism, which is also the rate-limiting step in generating the carboxylic end products. Saxagliptin cannot be hydrolyzed to carboxylic acid possibly because its amide cannot be formed in the first step.

To test whether other compounds containing amide groups could also be hydrolyzed by DPP-4, lacosamide, finasteride, niclosamide, bicalutamide, and flutamide were incubated with DPP-4. None of them could be metabolized to carboxylic acid metabolite (data not shown). The catalytic triad of human DPP-4 comprises Ser630, Asp708, and His740, which are located in a large cavity at the interface of the two domains of the protein (Thoma et al., 2003). To cyanopyrrolidine DPP-4 inhibitors, pyrrolidine was appended to an amino acid or the surrogate group to mimic proline, and the nitrile group was most likely covalently bound to Ser630 and within hydrogen bonding distance of Tyr547 (Oefner et al., 2003; Augeri et al., 2005). Thus, cyanopyrrolidine could bind to the active site of catalysis, providing the inhibitory activity and the potential of hydrolysis. Therefore, it is assumed that the precondition of the hydrolysis was that the nitrile group should enter the catalytic site of DPPs (Matteucci and Giampietro, 2009). The mechanism of nitrile
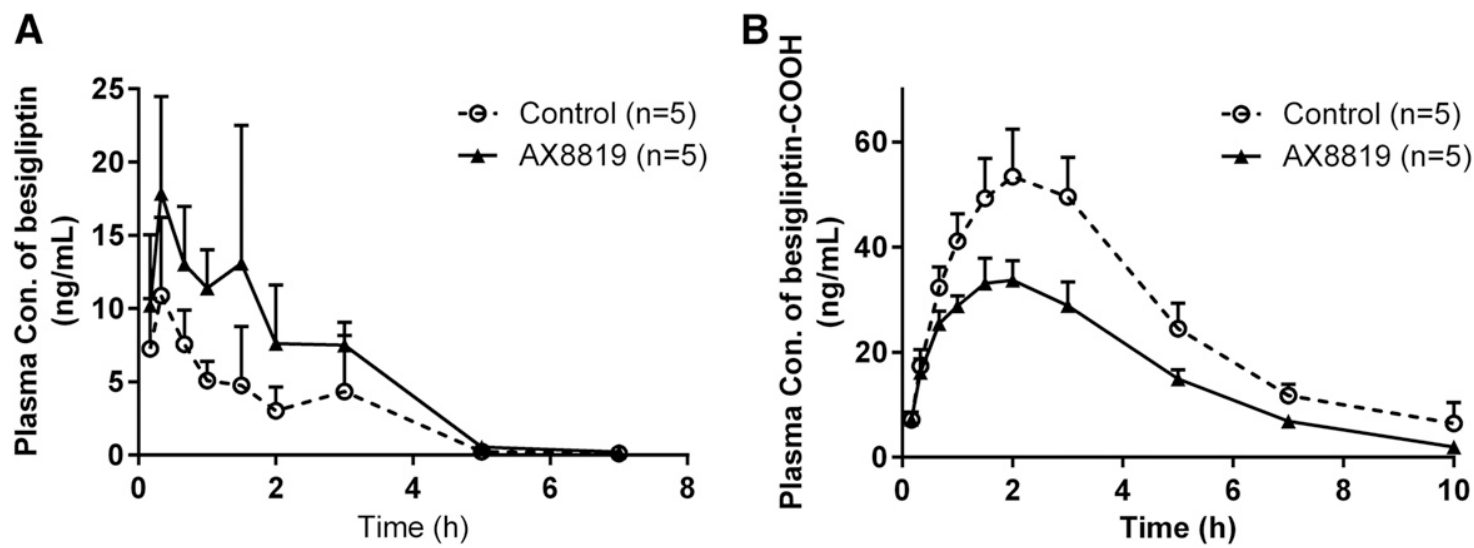

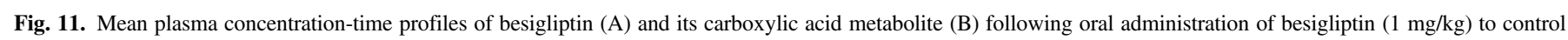
and AX8819-treated rats. 


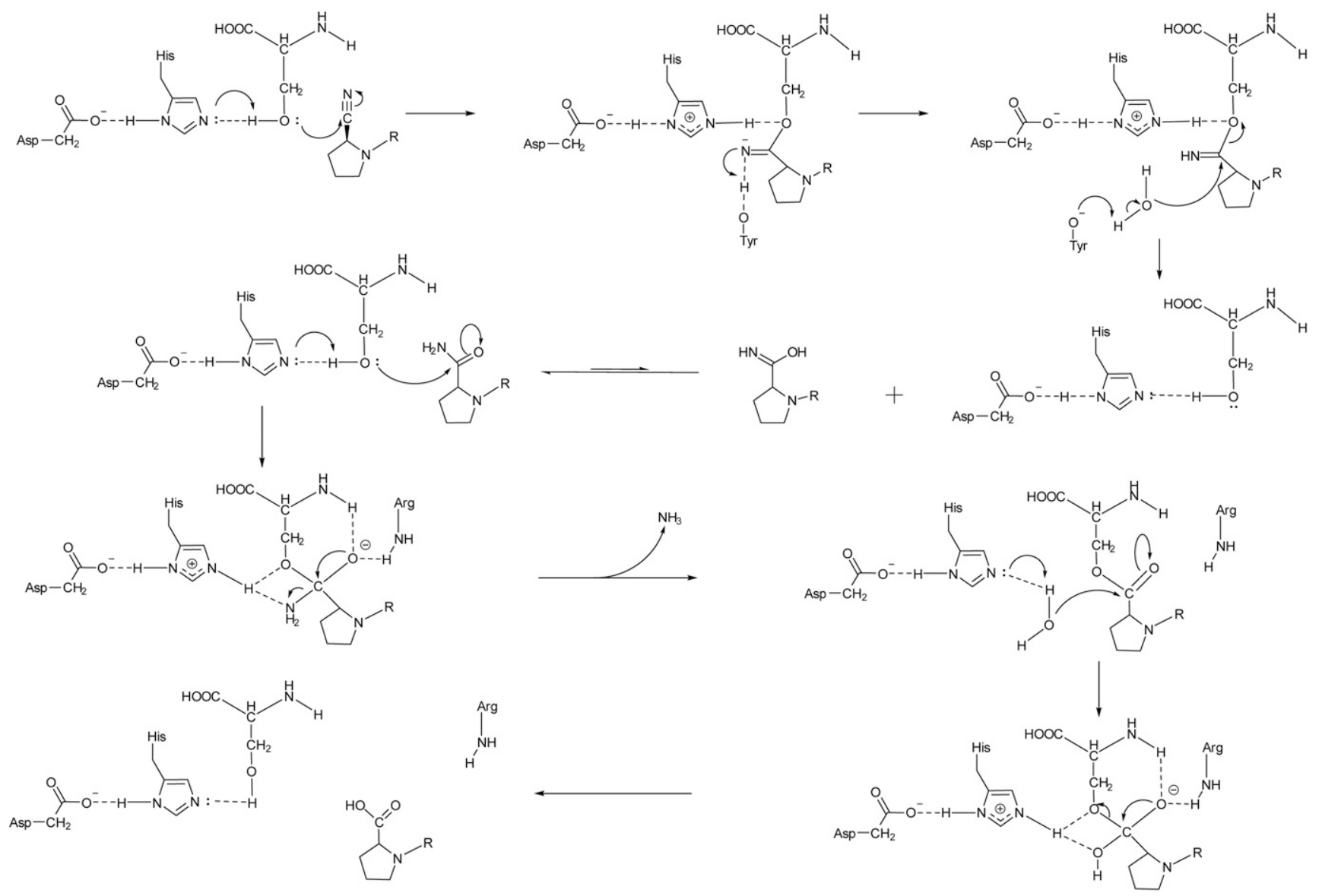

Fig. 12. Hydrolysis mechanism of nitrile group by the catalytic triad of Asp-His-Ser with the amide intermediate.

group hydrolysis by DPP-4 was speculated (Fig. 12). The serine protease catalysis included the acylation of the active site serine, subsequent release of the amine-leaving group, and hydrolysis of the acyl intermediate. The oxyanion of serine nucleophilic attacked nitrile carbon with the assistance from histidine. The electronegative nitrogen took the hydrogen of tyrosine and became imine. The first $\mathrm{H}_{2} \mathrm{O}$ participated, and the imine was bound with a hydroxyl, which was so unstable that it transformed to the amide intermediate (Kim et al., 2006). The amide intermediate still positioned in the catalytic triad of Asp-His-Ser by hydrogen bonding and was also attacked by the oxyanion of serine to form a tetrahedral intermediate. The next step was the reconstruction of the carbonyl double bond with the expulsion of the leaving ammonia. The second molecule of water took part in the restoration of the carbonyl double bond, and finally, the catalytic triad was released to the beginning state. However, for DPP2 , the hydrolysis mechanism may be different because two molecules of water entered into the active site in one step. Therefore, the amide intermediate was hydrolyzed to the carboxylic acid more rapidly, such that the number of carboxylic end products was higher than that of the amide intermediate (Fig. 4).

In conclusion, this study found that besides DPP-4, cyanopyrrolidine gliptins could also be hydrolyzed to the amide metabolites and end-product carboxylic acid metabolites by other serine peptidases in the DPP family, especially DPP-2. The effects of the aforementioned DPPs and their contribution to metabolism in vivo should be further evaluated. The study may also provide some useful information for drug design and metabolism investigation of nitrilecontaining compounds.

\section{Acknowledgments}

We greatly appreciate Dr. Youhong Hu (Shanghai Institute of Materia Medica, Chinese Academy of Sciences) for the synthesis of AX8819 and Dr. Jingya Li (National Center for Drug Screening, Shanghai Institute of Materia Medica, Chinese Academy of Sciences) for kindly providing recombinant DPPs.

\section{Authorship Contributions}

Participated in research design: Kong, Pang, Chen.

Conducted experiments: Kong, Pang, Deng.

Contributed new reagents or analytic tools: Kong, Pang, Zhong, Chen.

Performed data analysis: Kong, Pang, Zhao, Zheng, Chen.

Wrote or contributed to the writing of the manuscript: Kong, Pang, Chen.

\section{References}

Asakura M, Fujii H, Atsuda K, Itoh T, and Fujiwara R (2015) Dipeptidyl peptidase-4 greatly contributes to the hydrolysis of vildagliptin in human liver. Drug Metab Dispos 43 477-484.

Augeri DJ, Robl JA, Betebenner DA, Magnin DR, Khanna A, Robertson JG, Wang A, Simpkins LM, Taunk P, Huang Q, et al. (2005) Discovery and preclinical profile of saxagliptin (BMS-477118): a highly potent, long-acting, orally active dipeptidyl peptidase IV inhibitor for the treatment of type 2 diabetes. $J$ Med Chem 48:5025-5037.

Danilova O, Li B, Szardenings AK, Huber BT, and Rosenblum JS (2007) Synthesis and activity of a potent, specific azabicyclo[3.3.0]-octane-based DPP II inhibitor. Bioorg Med Chem Lett 17: 507-510.

Durinx C, Lambeir AM, Bosmans E, Falmagne JB, Berghmans R, Haemers A, Scharpé S, and De Meester I (2000) Molecular characterization of dipeptidyl peptidase activity in serum: soluble CD26/dipeptidyl peptidase IV is responsible for the release of X-Pro dipeptides. Eur J Biochem 267:5608-5613.

Fagerberg L, Hallström BM, Oksvold P, Kampf C, Djureinovic D, Odeberg J, Habuka M, Tahmasebpoor S, Danielsson A, Edlund K, et al. (2014) Analysis of the human tissue-specific expression by genomewide integration of transcriptomics and antibody-based proteomics. Mol Cell Proteomics 13:397-406.

Fleming FF, Yao L, Ravikumar PC, Funk L, and Shook BC (2010) Nitrile-containing pharmaceuticals: efficacious roles of the nitrile pharmacophore. J Med Chem 53:7902-7917. 
Furuta S, Smart C, Hackett A, Benning R, and Warrington S (2013) Pharmacokinetics and metabolism of $\left[{ }^{14} \mathrm{C}\right]$ anagliptin, a novel dipeptidyl peptidase-4 inhibitor, in humans. Xenobiotica 43: 432-442.

Gautier JF, Fetita S, Sobngwi E, and Salaün-Martin C (2005) Biological actions of the incretins GIP and GLP-1 and therapeutic perspectives in patients with type 2 diabetes. Diabetes Metab 31: 233-242.

Harstad EB, Rosenblum JS, Gorrell MD, Achanzar WE, Minimo L, Wu J, Rosini-Marthaler L, Gullo R, Ordway ND, Kirby MS, et al. (2013) DPP8 and DPP9 expression in cynomolgus monkey and Sprague Dawley rat tissues. Regul Pept 186:26-35.

He H, Tran P, Yin H, Smith H, Batard Y, Wang L, Einolf H, Gu H, Mangold JB, Fischer V, et al (2009a) Absorption, metabolism, and excretion of $\left[{ }^{14} \mathrm{C}\right]$ vildagliptin, a novel dipeptidyl peptidase 4 inhibitor, in humans. Drug Metab Dispos 37:536-544.

He H, Tran P, Yin H, Smith H, Flood D, Kramp R, Filipeck R, Fischer V, and Howard D (2009b) Disposition of vildagliptin, a novel dipeptidyl peptidase 4 inhibitor, in rats and dogs. Drug Metab Dispos 37:545-554.

Kim YB, Kopcho LM, Kirby MS, Hamann LG, Weigelt CA, Metzler WJ, and Marcinkeviciene J (2006) Mechanism of Gly-Pro-pNA cleavage catalyzed by dipeptidyl peptidase-IV and its inhibition by saxagliptin (BMS-477118). Arch Biochem Biophys 445:9-18.
Matteucci E and Giampietro O (2009) Dipeptidyl peptidase-4 (CD26): knowing the function before inhibiting the enzyme. Curr Med Chem 16:2943-2951.

Oefner C, D'Arcy A, Mac Sweeney A, Pierau S, Gardiner R, and Dale GE (2003) High-resolution structure of human apo dipeptidyl peptidase IV/CD26 and its complex with 1-[([2-[(5-iodopyridin2-yl)amino]-ethyl]amino)-acetyl]-2-cyano-(S)-pyrrolidine. Acta Crystallogr D Biol Crystallogr 59: 1206-1212.

Su H, Boulton DW, Barros A Jr., Wang L, Cao K, Bonacorsi SJ Jr., Iyer RA, Humphreys WG, and Christopher LJ (2012) Characterization of the in vitro and in vivo metabolism and disposition and cytochrome P450 inhibition/induction profile of saxagliptin in human. Drug Metab Dispos 40:1345-1356.

Thoma R, Löffler B, Stihle M, Huber W, Ruf A, and Hennig M (2003) Structural basis of prolinespecific exopeptidase activity as observed in human dipeptidyl peptidase-IV. Structure 11 $947-959$.

Address correspondence to: Dr. Xiaoyan Chen, Shanghai Institute of Materia Medica, Chinese Academy of Sciences, 501 Haike Road, Shanghai 201203, P.R. China. E-mail: xychen@simm.ac.cn 